\title{
Article \\ Generalized Rough Sets via Quantum Implications on Quantum Logic
}

\author{
Songsong Dai
}

School of Electronics and Information Engineering, Taizhou University, Taizhou 318000, China; ssdai@tzc.edu.cn

check for

updates

Citation: Dai, S. Generalized Rough Sets via Quantum Implications on Quantum Logic. Axioms 2022, 11, 2. https://doi.org/10.3390/axioms 11010002

Academic Editors: Alexander Šostak, Michal Holcapek, Antonin Dvorak and Amit K. Shukla

Received: 6 November 2021 Accepted: 21 December 2021 Published: 22 December 2021

Publisher's Note: MDPI stays neutral with regard to jurisdictional claims in published maps and institutional affiliations.

Copyright: (C) 2021 by the author. Licensee MDPI, Basel, Switzerland. This article is an open access article distributed under the terms and conditions of the Creative Commons Attribution (CC BY) license (https:// creativecommons.org/licenses/by/ $4.0 /)$.

\begin{abstract}
This paper introduces some new concepts of rough approximations via five quantum implications satisfying Birkhoff-von Neumann condition. We first establish rough approximations via Sasaki implication and show the equivalence between distributivity of multiplication over join and some properties of rough approximations. We further establish rough approximations via other four quantum implication and examine their properties.
\end{abstract}

Keywords: rough set; quantum logic; orthomodular lattice; quantum implication; Sasaki implication

MSC: 03G12; 06D72

\section{Introduction}

In 1982, aiming to give a mathematical tool for incomplete information processing, Pawlak [1] introduced the theory of rough sets. This theory has been widely used in many fields. The key of rough set is a pair of operators called lower and upper approximation operators. Many scholars have generalized the notion of rough approximation operators in different way. One way is to define these operators in different mathematical structures, such as modal logics [2-4], topological structures [5,6], Boolean algebra [7,8], lattice effect algebra [9], residuated lattices [10-15], and others [16-18].

Quantum computers were first introduced by Feynman $[19,20]$ and formalized by Deutsch [21]. Shor [22] gave a polynomial-time quantum algorithm for factoring integers in 1994 and Grover [23] introduced a quantum algorithm for unstructured searching in 1996. Their works greatly stimulated the research of quantum computation. With the advent of quantum computation, it is natural to ask the question: how to use the rough sets method in quantum computation and vice versa. Our method is from a logical point of view. Since quantum computation is a beautiful combination of quantum theory and computer science. As early as in 1936, in order to give a logic of quantum mechanics, Birkhoff and von Neumann [24] introduced quantum logic, whose algebraic model is an orthomodular lattice. Then, the issue is how to apply quantum logic in the analysis and design of rough sets. In the recent years, some scholars studied rough sets based on quantum logic. In 2017, Hassan [25] showed that rough set model with quantum logic can be used for recognition and classification systems. In our previous work [26,27], we proposed a rough set model based on quantum logic. We defined rough approximation operators via join and meet on a complete orthomodular lattice (COL). Some properties in our previous work are based on the distributivity of meet over join. However, any orthomodular lattice satisfying distributivity of meet over join reduces to a Boolean algebra. Moreover, some straightforward equivalences between distributivity and properties are proved. This means that these properties of rough sets theory hold if, and only if, the orthomodular lattice is a Boolean algebra. So these properties of rough sets theory hold in the frame of classical logic and may not hold in the frame of quantum logic. It is necessary to consider other rough sets model based on quantum logic, making more properties of rough sets hold in the frame of quantum logic. Quantum implication operators are important in the study of quantum logic. For example, they can be used to define deduction rules in 
quantum reasoning. This paper, therefore, discusses the quantum rough approximation operators based on quantum implications.

The paper is organized as follows: Section 2, we recall the concepts of orthomodular lattices. Since there are five quantum implications satisfying Birkhoff-von Neumann condition. Section 3, we redefine the rough approximation operators via the multiplication and Sasaki implication. In Section 4, we introduce the rough approximation operators via other four quantum implications. The conclusion is given in the final section.

\section{Preliminaries}

\subsection{Quantum Implicator}

First, we recall the concept of COL and its implicators [28-34].

A COL $L=<\mathbf{L}, \leq, \wedge, \vee, \perp, 0,1>$ is a complete bounded lattice with a unary operator $\perp$ which has the following properties: for all $u, v \in \mathbf{L}$

(C1) $u^{\perp} \vee u=1, u^{\perp} \wedge u=0$;

(C2) $u^{\perp \perp}=u$;

(C3) $u \leq v \Rightarrow v^{\perp} \leq u^{\perp}$;

(C4) $u \geq v \Rightarrow u \wedge\left(u^{\perp} \vee v\right)=v$.

where 0 and 1 are the least and greatest elements of $\mathbf{L}, \leq$ is the partial ordering in $\mathbf{L}, u \wedge v$ and $u \vee v$ stand for the greatest lower bound and the least upper bound of $u$ and $v$.

Quantum logic is a COL-valued logic and classical logic is treated as a Boolean algebra. The former is weaker than the latter. For example, the distributivity of meet over join holds in Boolean algebra, i.e., for all $u, v, w \in \mathbf{L}$,

$$
(v \wedge u) \vee(w \wedge u)=(v \vee w) \wedge u .
$$

However, it is not valid in a COL.

Implication operators in quantum logic can be defined in terms of $\perp, \vee$, and $\wedge$. They are required to satisfy the Birkhoff-von Neumann condition [24]: for any $u, v \in L$, $u \rightarrow v=1$ if, and only if, $u \leq v$. There are only five implication operators satisfying this condition $[35,36]$ :

Sasaki implication:

$$
u \rightarrow_{1} v=u^{\perp} \vee(u \wedge v)
$$

Dishkant implication:

$$
u \rightarrow_{2} v=v \vee\left(u^{\perp} \wedge v^{\perp}\right)
$$

Kalmbach implication:

$$
u \rightarrow_{3} v=\left(u^{\perp} \wedge v\right) \vee(u \wedge v) \vee\left(u^{\perp} \wedge v^{\perp}\right)
$$

Non-tollens implication:

$$
u \rightarrow_{4} v=\left(u^{\perp} \wedge v\right) \vee(u \wedge v) \vee\left(\left(u^{\perp} \vee v\right) \wedge v^{\perp}\right)
$$

Relevance implication:

$$
u \rightarrow_{5} v=\left(u^{\perp} \wedge v\right) \vee\left(u^{\perp} \wedge v^{\perp}\right) \vee\left(u \wedge\left(u^{\perp} \vee v\right)\right)
$$

Moreover, the multiplication operator is defined as follows: for all $u, v \in L$,

$$
u \& v={ }_{\operatorname{def}}\left(u \vee v^{\perp}\right) \wedge v .
$$

Remark 1. For any $u, v \in L, u \rightarrow_{2} v=v^{\perp} \rightarrow_{1} u^{\perp}, u \rightarrow_{4} v=v^{\perp} \rightarrow_{3} u^{\perp}$.

Remark 2. If $L$ is a Boolean algebra, $u \rightarrow_{i} v(i=1,2, \ldots, 5)$ is equivalent to $u \rightarrow_{0} v=u^{\perp} \vee v$ which is named "material implication". 
Remark 3. For any orthomodular lattice, among $\rightarrow_{i}(i=1,2,3,4,5)$, Sasaki implication $\rightarrow_{1}$ is unique one satisfying the following condition $[37,38]$ : there exists binary operation and such that for any $u, v, w \in L, u \& v \leq w$ if, and only if, $u \leq v \rightarrow_{1} w$.

The following are some properties of the Sasaki implication and the multiplication:

(C5) $u \& v \leq w$ iff $u \leq v \rightarrow w$;

(C6) $0 \& u=0, u \& 1=u, 1 \rightarrow u=u$ and $u \rightarrow 1=1$;

(C7) $u \wedge v \leq u \& v$;

(C8) $u \leq v$ if, and only if, $u \rightarrow v=1$

Let $l=<L, \leq, \wedge, \vee, \perp, 0,1>$ be a COL, then $L$ is a Boolean algebra, if, and only if, any one of the following condition holds:

(C9) \& is commutative, i.e., $u \& v=v \& u$ for any $u, v \in L$;

(C10) $v \leq w \Rightarrow u \& v \leq u \& w$ for any $u, v, w \in L$.

\subsection{Dual Operator of Quantum Implicator}

Based on $\perp$, a dual operator $\hookrightarrow_{i}$ of quantum implicator $\rightarrow_{i}$ is defined as follow: for all $u, v \in L$,

$$
u \hookrightarrow_{i} v=\left(u^{\perp} \rightarrow_{i} v^{\perp}\right)^{\perp} .
$$

Proposition 1. All five operator $\vartheta_{i}(i=1,2, \ldots, 5)$ satisfy the condition: for any $u, v \in L, v \leq u$ if, and only if, $u \hookrightarrow_{i} v=0$.

Proof. It can be deduced from the following, for any $u, v \in L$,

$$
\begin{aligned}
v \leq u & \Leftrightarrow u^{\perp} \leq v^{\perp} \\
& \Leftrightarrow u^{\perp} \rightarrow_{i} v^{\perp}=1 \\
& \Leftrightarrow\left(u^{\perp} \rightarrow_{i} v^{\perp}\right)^{\perp}=0 \\
& \Leftrightarrow u \hookrightarrow_{i} v=0 .
\end{aligned}
$$

Proposition 2. For any $u, v \in L, v^{\perp} \hookrightarrow_{1} u=u \& v$

Proof. It can be deduced from the following,

$$
\begin{aligned}
v^{\perp} \hookrightarrow_{1} u & =\left(v \rightarrow_{1} u^{\perp}\right)^{\perp} \\
& =\left(v^{\perp} \vee\left(v \wedge u^{\perp}\right)\right)^{\perp} \\
& =v \wedge\left(v^{\perp} \vee u\right) \\
& =u \& v .
\end{aligned}
$$

The bi-implication operator corresponding to the Sasaki implication is defined as follows: for any $u, v \in L$,

$$
u \leftrightarrow v=\operatorname{def}(u \rightarrow v) \wedge(v \rightarrow u)
$$

Clearly, $u=v$ if, and only if, $u \leftrightarrow v=1$.

Let $X$ be a finite set, $L$ a COL, $E$ a binary relation on $X$ relative to $L$. Then,

$E$ is serial if for all $u \in X, \vee_{v \in X} E(u, v)=1$.

$E$ is reflexive if $E(u, u)=1$ holds for all $u \in X$.

$E$ is symmetric if $E(u, v)=E(v, u)$ holds for all $u, v \in X$.

$E$ is \&-transitive if $E(u, w) \geq \vee_{v \in X} E(v, w) \& E(u, v)$ holds for all $u, v, w \in X$. 


\section{Rough Approximations via Sasaki Implication $\rightarrow_{1}$ and Multiplication \&}

In this section, the new rough approximations are defined by using the multiplication connective \& and Sasaki implication $\rightarrow_{1}$. For convenience, we use $\rightarrow$ in place of $\rightarrow_{1}$ in this section.

Definition 1. Let $U$ be a finite set, $L$ a $C O L, E$ a binary relation on $U$ relative to $L$, and $X a$ l-valued set in $U$. A pair of lower and upper rough approximations of $X, E_{\&} X$ and $\bar{E}_{\&} X$, are defined, respectively, as follows:

$$
\left(\underline{E}_{\&} X\right)(x)=\bigwedge_{y \in U}(E(x, y) \rightarrow X(y)), \quad \forall x \in U
$$

and

$$
\left(\bar{E}_{\&} X\right)(x)=\bigvee_{y \in U}(X(y) \& E(x, y)), \quad \forall x \in U
$$

In our previous work [26], rough approximations are defined based on $\wedge$ and $\vee$, i.e.,

$$
\begin{aligned}
& \left(\underline{E}_{\wedge} X\right)(x)=\bigwedge_{y \in U}\left(E(x, y)^{\perp} \vee X(y)\right), \forall x \in U \\
& \left(\bar{E}_{\wedge} X\right)(x)=\bigvee_{y \in U}(X(y) \wedge E(x, y)), \forall x \in U .
\end{aligned}
$$

Clearly, we have $\left(\bar{E}_{\wedge} X\right)(x) \leq\left(\bar{E}_{\&} X\right)(x)$ because $u \wedge v \leq u \& v, \forall u, v \in L$.

Remark 4. In [26,27], we gave some results of $\left(\underline{E}_{\wedge} X\right)$ and $\left(\bar{E}_{\wedge} X\right)$ rely on the distributivity of meet over join. For example, $\bar{E}_{\&}(X \cup Y) \equiv\left(\bar{E}_{\&} X \cup \bar{E}_{\&} Y\right)$ is equivalent to $(v \& u) \vee(w \& u)=$ $(v \vee w) \& u, \forall u, v, w \in L$. This is indeed a negative result since any orthomodular lattice satisfying distributivity of meet over join reduces to a Boolean algebra.

Remark 5. A complete orthomodular lattice $L$ is a Boolean algebra if, and only if, \& is commutative, so $X(y) \& E(x, y)$ and $E(x, y) \& X(y)$ are different.

Example 1. Consider the smallest orthomodular lattice which is not a Boolean algebra, called MO2 [39], as given in Figure 1. Let the universe $U=\{x, y\}$. Define a L-valued set

$$
X=\frac{u}{x}+\frac{v}{y}
$$

and a L-valued relation $R$ on MO2 in Table 1. Then, by the work in [26], we have

Table 1. The $L$-valued relation $E$ in Example 1.

\begin{tabular}{lll}
\hline $\mathbf{R}$ & $x$ & $y$ \\
\hline$x$ & 1 & 0 \\
$y$ & 0 & $\mathrm{u}$ \\
\hline
\end{tabular}




$$
\begin{aligned}
\left(\underline{E}_{\wedge} X\right)(x) & =\left(E(x, x)^{\perp} \vee X(x)\right) \bigwedge\left(E(x, y)^{\perp} \vee X(y)\right) \\
& =\left(1^{\perp} \vee u\right) \wedge\left(0^{\perp} \vee v\right) \\
& =u \wedge 1=u \\
\left(\underline{E}_{\wedge} X\right)(y) & =\left(E(y, x)^{\perp} \vee X(x)\right) \wedge\left(E(y, y)^{\perp} \vee X(y)\right) \\
& =\left(0^{\perp} \vee u\right) \wedge\left(u^{\perp} \vee v\right) \\
& =1 \wedge 1=1 \\
\left(\bar{E}_{\wedge} X\right)(x) & =(E(x, x) \wedge E(x)) \bigvee(E(x, y) \wedge E(y)) \\
& =(1 \wedge u) \bigvee(0 \wedge v) \\
& =u \bigvee 0=u \\
\left(\bar{E}_{\wedge} X\right)(y) & =(E(y, x) \wedge E(x)) \bigvee(E(y, y) \wedge E(y)) \\
& =(0 \wedge u) \bigvee(u \wedge v) \\
& =0 \bigvee 0=0
\end{aligned}
$$

Thus, we obtain

$$
\begin{aligned}
& \underline{E}_{\wedge} X=\frac{u}{x}+\frac{1}{y} \\
& \bar{E}_{\wedge} X=\frac{u}{x}+\frac{0}{y}
\end{aligned}
$$

By Equations (5) and (6), we have

$$
\begin{aligned}
\left(\underline{E}_{\&} X\right)(x) & =(E(x, x) \rightarrow X(x)) \bigwedge(E(x, y) \rightarrow X(y)) \\
& =(1 \rightarrow u) \bigwedge(0 \rightarrow v) \\
& =u \bigwedge 1=u \\
\left(\underline{E}_{\&} X\right)(y) & =(E(y, x) \rightarrow X(x)) \bigwedge(E(y, y) \rightarrow X(y)) \\
& =(0 \rightarrow u) \bigwedge(u \rightarrow v) \\
& =1 \bigwedge u^{\perp}=u^{\perp} \\
\left(\bar{E}_{\&} X\right)(x) & =(X(x) \& E(x, x)) \bigvee(X(y) \& E(x, y)) \\
& =(u \& 1) \bigvee(v \& 0) \\
& =u \bigvee 0=u \\
\left(\bar{E}_{\&} X\right)(y) & =(X(x) \& E(y, x)) \bigvee(X(y) \& E(y, y)) \\
& =(u \& 0) \bigvee(v \& u) \\
& =0 \bigvee u=u
\end{aligned}
$$

Thus, we obtain

$$
\begin{array}{r}
\underline{E}_{\&} X=\frac{u}{x}+\frac{u^{\perp}}{y} \\
\bar{E}_{\&} X=\frac{u}{x}+\frac{u}{y}
\end{array}
$$

Clearly, $\underline{E}_{\wedge} X \neq \underline{E}_{\&} X$ and $\bar{E}_{\wedge} X \neq \bar{E}_{\&} X$. 


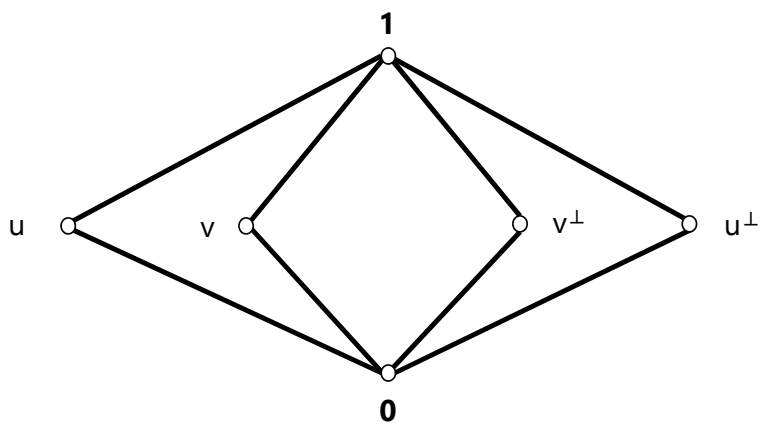

Figure 1. Orthomodular lattice MO2 [39].

Proposition 3. Let $U$ be a finite set, $L$ a COL, E a binary relation on $U$ relative to $L$, and $X$ a $l$-valued set in $U$. Then,

(1) $\underline{E}_{\&} \varnothing \equiv \varnothing, \bar{E}_{\&} U \equiv U$;

(2) $\underline{E}_{\&} X^{\perp} \equiv\left(\bar{E}_{\&} X\right)^{\perp}$ and $\bar{E}_{\&} X^{\perp} \equiv\left(\underline{E}_{\&} X\right)^{\perp}$;

(3) $X \subseteq \bar{E}_{\&} X$, if, and only if, $\underline{E}_{\&} X \subseteq X$, if, and only if, $E$ is reflexive;

(4) $\bar{E}_{\wedge} X \subseteq \bar{E}_{\&} X$ and $\underline{E} X_{\&} \subseteq \underline{E}_{\wedge} X$;

(5) If $X \subseteq Y$, then $\underline{E}_{\&} X \subseteq \underline{E}_{\&} Y$ and $\bar{E}_{\&} X \subseteq \bar{E}_{\&} Y$;

(6) $\left(\underline{E}_{\&} X \cup \underline{E}_{\&} Y\right) \subseteq \underline{E}_{\&}(X \cup Y),\left(\bar{E}_{\&}(X \cap Y) \subseteq\left(\bar{E}_{\&} X \cap \bar{E}_{\&} Y\right)\right.$;

(7) $\bigcup_{i \in J} \bar{E}_{\&} X_{i} \subseteq \bar{E}_{\&}\left(\bigcup_{i \in J} X_{i}\right)$ and $\underline{E}_{\&}\left(\bigcap_{i \in J} X_{i}\right) \subseteq \bigcap_{i \in J} \underline{E}_{\&} X_{i}$ for any $X_{i} \in L^{U}, i \in J$.

Proof. (1) and (3) From (C6), i.e., $0 \& u=0, u \& 1=u, 1 \rightarrow u=u$, and $u \rightarrow 1=1$;

(2) From $(u \rightarrow v)^{\perp}=\left(u^{\perp} \vee(u \wedge v)\right)^{\perp}=u \wedge\left(v^{\perp} \vee u^{\perp}\right)=v^{\perp} \& u$ for any $u, v \in L$;

(4) From (C7), i.e., $u \wedge v \leq u \& v$;

(5-7) For any $u, v, w \in L v \leq w \Rightarrow v \& u \leq w \& u$ for any $u, v, w \in L$.

Proposition 4. For any binary relation $E$ and l-valued set $X$ on $U$,

$$
\begin{aligned}
& \bar{E}_{\&} X=\left(\underline{E}_{\&} X^{\perp}\right)^{\perp}, \\
& \underline{E}_{\&} X=\left(\bar{E}_{\&} X^{\perp}\right)^{\perp} .
\end{aligned}
$$

Proof. Since for any $u, v \in L$, we have $\left(v \rightarrow u^{\perp}\right)^{\perp}=\left(v^{\perp} \vee\left(v \wedge u^{\perp}\right)\right)^{\perp}=v \wedge\left(v^{\perp} \vee u\right)=$ $u \& v$.

Example 2. See Example 1, we have

$$
\begin{aligned}
\left(\underline{E}_{\&} X^{\perp}\right)^{\perp}(x) & =\left(\left(E(x, x) \rightarrow X^{\perp}(x)\right) \bigwedge\left(E(x, y) \rightarrow X^{\perp}(y)\right)\right) \\
& =\left(\left(1 \rightarrow u^{\perp}\right) \bigwedge\left(0 \rightarrow v^{\perp}\right)\right)^{\perp} \\
& =\left(u^{\perp} \bigwedge 1\right)^{\perp} \\
& =u \bigvee 0=u \\
\left(\underline{E}_{\&} X^{\perp}\right)^{\perp}(y) & =\left(\left(E(y, x) \rightarrow X^{\perp}(x)\right) \bigwedge\left(E(y, y) \rightarrow X^{\perp}(y)\right)\right)^{\perp} \\
& =\left(\left(0 \rightarrow u^{\perp}\right) \bigwedge\left(u \rightarrow v^{\perp}\right)\right)^{\perp} \\
& =\left(1 \bigwedge u^{\perp}\right)^{\perp} \\
& =0 \bigvee u=u
\end{aligned}
$$




$$
\begin{aligned}
\left(\bar{E}_{\&} X^{\perp}\right)^{\perp}(x) & =\left(\left(X^{\perp}(x) \& E(x, x)\right) \bigvee\left(X^{\perp}(y) \& E(x, y)\right)\right)^{\perp} \\
& =\left(\left(u^{\perp} \& 1\right) \bigvee\left(v^{\perp} \& 0\right)\right)^{\perp} \\
& =\left(u^{\perp} \bigvee 0\right)^{\perp} \\
& =u \bigwedge 1=u \\
\left(\bar{E}_{\&} X^{\perp}\right)^{\perp}(y) & =\left(\left(X^{\perp}(x) \& E(y, x)\right) \bigvee\left(X^{\perp}(y) \& E(y, y)\right)\right)^{\perp} \\
& =\left(\left(u^{\perp} \& 0\right) \bigvee\left(v^{\perp} \& u\right)\right)^{\perp} \\
& =(0 \bigvee u)^{\perp} \\
& =1 \bigwedge u^{\perp}=u^{\perp}
\end{aligned}
$$

It is easy to verify that $\left(\bar{E}_{\&} X^{\perp}\right)^{\perp}=\underline{E}_{\&} X$ and $\left(\underline{E}_{\&} X^{\perp}\right)^{\perp}=\bar{E}_{\&} X$.

Proposition 5. The following three statements are equivalent:

(1) For any $u, v, w \in L,(v \& u) \vee(w \& u)=(v \vee w) \& u$;

(2) $\bar{E}_{\&}(X \cup Y) \equiv\left(\bar{E}_{\&} X \cup \bar{E}_{\&} Y\right)$;

(3) $\underline{E}_{\&}(X \cap Y) \equiv\left(\underline{E}_{\&} X \cap \underline{E}_{\&} Y\right)$.

Proof. $(1) \Rightarrow(2)$ : By using the distributive law of \& over $\vee, U$ is a finite set. We have

$$
\begin{aligned}
\bar{E}_{\&}(X \vee Y)(x) & =\bigvee_{y \in U}((X \vee Y)(y) \& E(x, y)) \\
& =\bigvee_{y \in U}((X(y) \vee Y(y)) \& E(x, y)) \\
& =\bigvee_{y \in U}((X(y) \& E(x, y)) \vee(Y(y) \& E(x, y))) \\
& =\left(\bar{E}_{\&} X \vee \bar{E}_{\&} Y\right)(x) .
\end{aligned}
$$

(2) $\Rightarrow(1)$ : Given $u, v, w \in L$, then the purpose is to show $(v \& u) \vee(w \& u)=(v \vee w) \& u$ for any $u, v, w \in L$. Let $E\left(x, y_{1}\right)=u$, and $E(x, y)=0$ for other $y \in U ; X\left(y_{1}\right)=v, Y\left(y_{1}\right)=w$, and $X(y)=Y(y)=0$ for other $y \in U$. Then, we have

$$
\begin{aligned}
\bar{E}_{\&}(X \vee Y)(x) & =\bigvee_{y \in U}((X \vee Y)(y) \& e(x, y)) \\
& =(X \vee Y)\left(y_{1}\right) \& E\left(x, y_{1}\right) \\
& =(v \vee w) \& u .
\end{aligned}
$$

and

$$
\begin{aligned}
\left(\bar{E}_{\&} X \vee \bar{E}_{\&} Y\right)(x) & =\bar{E}_{\&} X(x) \vee \bar{E}_{\&} Y(x) \\
& =\left(\bigvee_{y \in U}(X(y) \& E(x, y))\right) \vee\left(\bigvee_{y \in U}(Y(y) \& E(x, y))\right) \\
& =\left(X\left(y_{1}\right) \& E\left(x, y_{1}\right)\right) \vee\left(Y\left(y_{1}\right) \& E\left(x, y_{1}\right)\right) \\
& =(v \& u) \vee(w \& u)
\end{aligned}
$$

Therefore, $(v \vee w) \& u=(v \& u) \vee(w \& u)$. 
$(2) \Rightarrow(3)$, from Proposition 4 and (2),

$$
\begin{aligned}
\underline{E}_{\&}(X \cap Y) & =\left(\bar{E}_{\&}\left(X^{\perp} \cup Y^{\perp}\right)\right)^{\perp} \\
& =\left(\bar{E}_{\&} X^{\perp} \cup \bar{E}_{\&} Y^{\perp}\right)^{\perp} \\
& =\left(\underline{E}_{\&} X \cap \underline{E}_{\&} Y\right) .
\end{aligned}
$$

(3) $\Rightarrow(2)$, from Proposition 4 and (3),

$$
\begin{aligned}
\bar{E}_{\&}(X \cup Y) & =\left(\underline{E}_{\&}\left(X^{\perp} \cap Y^{\perp}\right)\right)^{\perp} \\
& =\left(\underline{E}_{\&} X^{\perp} \cap \underline{E}_{\&} Y^{\perp}\right)^{\perp} \\
& =\left(\bar{E}_{\&} X \cup \bar{E}_{\&} Y\right) .
\end{aligned}
$$

Proposition 6. If $L$ satisfies the distributivity of \& over $\vee$. Then, the following three statements are equivalent.

(1) E is serial;

(2) $\bar{E}_{\&} \hat{a} \equiv \hat{a}$, for any $a \in L$;

(3) $\underline{E}_{\&} \hat{a} \equiv \hat{a}$, for any $a \in L$.

where $\hat{a}(u)=a$, for any $u \in U$.

Proof. $(1) \Rightarrow(2)$ : By using the distributive law of \& over $\vee$, we have

$$
\begin{aligned}
\bar{E}_{\&} \hat{a}(u) & =\bigvee_{v \in U}(\hat{a}(v) \& E(u, v)) \\
& =\bigvee_{v \in U}(a \& E(u, v)) \\
& =a \& \bigvee_{v \in U} E(u, v) \\
& =a \& 1 \\
& =a .
\end{aligned}
$$

(2) $\Rightarrow(1)$ : Take $a=1$; then it follows from the proof of necessity and $\bar{E}_{\&} \hat{1}(u) \leftrightarrow 1(u)$ for every $u \in X$ that $\bigvee_{v \in U} E(u, v)=1$ holds for every $u \in X$. Hence $E$ is serial.

Similarly, we can prove (1) $\Leftrightarrow(3)$.

Example 3. Consider the following L-valued relation E on MO2, as given in Table 2. Define a $L$-valued set the universe $U=\{x, y\}$

$$
X=\frac{u}{x}+\frac{u}{y} .
$$

Table 2. The $L$-valued relation $E$ in Example 2.

\begin{tabular}{lll}
\hline $\mathbf{R}$ & $\boldsymbol{x}$ & $\mathrm{y}$ \\
\hline$x$ & $\mathrm{u}$ & $\mathrm{v}$ \\
$y$ & $\mathrm{v}$ & $\mathrm{u}$ \\
\hline
\end{tabular}


By Equations (5) and (6), we have

$$
\begin{aligned}
\left(\underline{E}_{\&} X\right)(x) & =(E(x, x) \rightarrow X(x)) \bigwedge(E(x, y) \rightarrow X(y)) \\
& =(u \rightarrow u) \bigwedge(v \rightarrow u) \\
& =1 \bigwedge v^{\perp}=v^{\perp} \\
\left(\underline{E}_{\&} X\right)(y) & =(E(y, x) \rightarrow X(x)) \bigwedge(E(y, y) \rightarrow X(y)) \\
& =(v \rightarrow u) \bigwedge(u \rightarrow u) \\
& =v^{\perp} \bigwedge 1=v^{\perp} \\
\left(\bar{E}_{\&} X\right)(x) & =(E(x) \& E(x, x)) \bigvee(E(y) \& E(x, y)) \\
& =(u \& u) \bigvee(u \& v) \\
& =u \bigvee v=1 \\
\left(\bar{E}_{\&} X\right)(y) & =(E(x) \& E(y, x)) \bigvee(E(y) \& E(y, y)) \\
& =(u \& v) \bigvee(u \& u) \\
& =v \bigvee u=1
\end{aligned}
$$

Thus, we obtain

$$
\begin{array}{r}
E_{\&} X=\frac{v^{\perp}}{x}+\frac{v^{\perp}}{y} \\
\bar{E}_{\&} X=\frac{1}{x}+\frac{1}{y} .
\end{array}
$$

$E$ is serial, but $L$ does not satisfy the distributivity of \& over $\vee$, so $\bar{E}_{\&} \hat{a} \neq \hat{a}$ and $\underline{E}_{\&} \hat{a} \neq \hat{a}$.

Proposition 7. If two of the following statements are hold, then the third statement holds:

(1) For any $u, v, w \in L,(v \& u) \vee(w \& u)=(v \vee w) \& u$;

(2) $E$ is \&-transitive;

(3) $\bar{E}_{\&}\left(\bar{E}_{\&} X\right) \subseteq \bar{E}_{\&} X$.

Proof. $(1)+(2) \Rightarrow(3)$,

$$
\begin{aligned}
\bar{E}_{\&}\left(\bar{E}_{\&} X\right)(x) & =\bigvee_{y \in U}\left(\bar{E}_{\&} X(y) \& E(x, y)\right) \\
& =\bigvee_{y \in U}\left(\left(\bigvee_{z \in U}(X(z) \& E(y, z)) \& E(x, y)\right)\right) \\
& =\bigvee_{z \in U}\left(X(z) \& \bigvee_{y \in U}(E(y, z) \& E(x, y))\right) \\
& \leq \bigvee_{z \in U}(X(z) \& E(x, z)) \\
& =\bar{E}_{\&} X(x) .
\end{aligned}
$$

$(1)+(3) \Rightarrow(2)$ : Assume that $E$ is not \&-transitive. It follows than that for some $u_{0}, w_{0} \in U$,

$$
\bigvee_{v \in U}\left(E\left(v, w_{0}\right) \& E\left(u_{0}, v\right)\right) \leq E\left(u_{0}, w_{0}\right)
$$

does not hold.

Let $X\left(w_{0}\right)=1$ and $X(v)=0$ for other $v \in U$. Then, we have

$$
\bar{E}_{\&}\left(\bar{E}_{\&} X\right)\left(u_{0}\right)=\bigvee_{v \in U}\left(E\left(v, w_{0}\right) \& E\left(u_{0}, v\right)\right)
$$


and

$$
\bar{E}_{\&} X\left(u_{0}\right)=E\left(u_{0}, w_{0}\right) .
$$

Therefore, it follows from (3) that

$$
\bigvee_{v \in U}\left(E\left(v, w_{0}\right) \& E\left(u_{0}, v\right)\right) \leq E\left(u_{0}, w_{0}\right)
$$

$(2)+(3) \Rightarrow(1)$ : Given $u, v, w \in L$, let $U=x, y, z, E \in L^{U \times U}$ which $E(x, y)=$ $E(x, z)=u, E(y, z)=E(z, z)=v, E(z, y)=E(y, y)=w$ and others are 0 , and $X \in L^{u}$ which $X(y)=w, X(z)=v, X(x)=0$. It easy to check that $E$ is transitive. Then,

$\bar{E}_{\&} X(x)=(X(y) \& E(x, y)) \vee(X(z) \& E(x, z))=(w \& u) \vee(v \& u)$

$\bar{E}_{\&} X(y)=(X(y) \& E(y, y)) \vee(X(z) \& E(y, z))=w \vee v$

$\bar{E}_{\&} X(z)=(X(z) \& E(z, z)) \vee(X(y) \& E(z, y))=w \vee v$

and

$$
\begin{aligned}
\bar{E}_{\&}\left(\bar{E}_{\&} X\right)(x) & =\left(\bar{E}_{\&} X(y) \& E(x, y)\right) \vee\left(\bar{E}_{\&} X(z) \& E(x, z)\right) \\
& =((v \vee w) \& u) \vee((v \vee w) \& u) \\
& =(v \vee w) \& u
\end{aligned}
$$

So by $\bar{E}_{\&}\left(\bar{E}_{\&} X\right)(x) \leq \bar{E}_{\&} X(x)$ we obtain $(v \vee w) \& u \leq((v \& u) \vee(w \& u))$.

Since $((v \vee w) \& u) \geq((v \& u) \vee(w \& u))$ always holds. Thus, we have $((v \vee w) \& u)=$ $((v \& u) \vee(w \& u))$.

Proposition 8. If two of the following statements are hold, then the third statement holds:

(1) For any $u, v, w \in L,(v \& u) \vee(w \& u)=(v \vee w) \& u$;

(2) $E$ is \&-transitive;

(3) $\underline{E}_{\&} X \subseteq \underline{E}_{\&}\left(\underline{E}_{\&} X\right)$.

Proof. Similar to that of Proposition 7.

Remark 6. For the proof of Propositions 7 and 8, in this paper, we use the concept that $E$ is \&-transitive if $E(u, w) \geq \vee_{v \in U} E(v, w) \& E(u, v)$ holds for all $u, v, w \in U$, not $E(u, w) \geq$ $\vee_{v \in U} E(u, v) \& E(v, w)$ holds for all $u, v, w \in U$.

Definition 2 ([34]). Let $U$ be a non-empty set and $L$ a COL, a function int: $L^{U} \rightarrow L^{U}$ is an $l$-valued interior operator if for all $G, H \in L^{U}$ it satisfies:
(1) $\operatorname{int}(\hat{a})=\hat{a}$;
(2) $\operatorname{int}(G) \subseteq G$;
(3) $\operatorname{int}(G \cap H)=\operatorname{int}(G) \cap \operatorname{int}(H)$;
(4) $\operatorname{int}(\operatorname{int}(G))=\operatorname{int}(G)$.

Definition 3 ([34]). Let $U$ be a non-empty set and $L$ a COL, a function $\mathrm{cl}: L^{U} \rightarrow L^{U}$ is an $l$-valued closure operator if for all $G, H \in L^{U}$ it satisfies:

(1) $\operatorname{cl}(\hat{a})=\hat{a}$;

(2) $G \subseteq \operatorname{cl}(G)$;

(3) $c l(G \cup H)=\operatorname{cl}(G) \cup \operatorname{cl}(H)$;

(4) $\operatorname{cl}(\operatorname{cl}(G))=\operatorname{cl}(G)$.

Proposition 9. If two of the following statements are hold, then the third statement holds:

(1) For any $u, v, w \in L,(v \& u) \vee(w \& u)=(v \vee w) \& u$;

(2) $E$ is serial, reflexive and \&-transitive;

(3) $\underline{E}_{\&}$ is an $l$-valued interior operator.

Proof. Immediate from Propositions 3, 5, 6, and 8. 
Proposition 10. If two of the following statements are hold, then the third statement holds:

(1) For any $u, v, w \in L,(v \& u) \vee(w \& u)=(v \vee w) \& u$;

(2) E is serial, reflexive and \&-transitive;

(3) $\bar{E}_{\&}$ is an $l$-valued closure operator.

Proof. Immediate from Propositions 3, 5, 6, and 7.

\section{Rough Approximations via Implicator $\rightarrow_{i}$ and Its Dual Operator $\hookrightarrow_{i}$}

For other quantum implication $\rightarrow_{i}(i=2, \ldots, 5)$, we use its dual operator $\hookrightarrow_{i}$ to define the upper rough approximation.

Definition 4. Let $U$ be a finite set, $L$ a $C O L, E$ a binary relation on $U$ relative to $L$, and $X$ a l-valued set in $U$. A pair of lower and upper rough approximations of $X, \underline{E}_{i} X$ and $\bar{E}_{i} X$, are defined, respectively, as follows:

$$
\left(\underline{E}_{i} X\right)(x)=\bigwedge_{y \in U}\left(E(x, y) \rightarrow_{i} X(y)\right), \quad \forall x \in U
$$

and

$$
\left(\bar{E}_{i} X\right)(x)=\bigvee_{y \in U}\left(E(x, y)^{\perp} \hookrightarrow_{i} X(y)\right), \quad \forall x \in U
$$

Example 4. Consider the orthomodular lattice MO2 [39], as given in Figure 1. Let the universe $U=\{x, y\}$. Define a L-valued set

$$
X=\frac{u}{x}+\frac{v}{y}
$$

and a L-valued relation $R$ on MO2 in Table 1. Then, by Equations (24) and (25), we have

$$
\begin{aligned}
\left(\underline{E}_{2} X\right)(x) & =\left(E(x, x) \rightarrow_{2} X(x)\right) \bigwedge\left(E(x, y) \rightarrow_{2} X(y)\right) \\
& =\left(1 \rightarrow_{2} u\right) \bigwedge\left(0 \rightarrow_{2} v\right) \\
& =u \bigwedge 1=u \\
\left(\underline{E}_{2} X\right)(y) & =\left(E(y, x) \rightarrow_{2} X(x)\right) \bigwedge\left(E(y, y) \rightarrow_{2} X(y)\right) \\
& =\left(0 \rightarrow_{2} u\right) \bigwedge\left(u \rightarrow_{2} v\right) \\
& =1 \bigwedge v=v \\
\left(\bar{E}_{2} X\right)(x) & =\left(E(x, x)^{\perp} \hookrightarrow_{2} E(x)\right) \bigvee\left(E(x, y)^{\perp} \hookrightarrow_{2} E(y)\right) \\
& =\left(1^{\perp} \hookrightarrow_{2} u\right) \bigvee\left(0^{\perp} \hookrightarrow_{2} v\right) \\
& =u \bigvee 0=u \\
\left(\bar{E}_{2} X\right)(y) & =\left(E(y, x)^{\perp} \hookrightarrow_{2} E(x)\right) \bigvee\left(E(y, y)^{\perp} \hookrightarrow_{2} E(y)\right) \\
& =\left(0^{\perp} \hookrightarrow_{2} u\right) \bigvee\left(u^{\perp} \hookrightarrow_{2} v\right) \\
& =0 \bigvee v=v
\end{aligned}
$$

Thus, we obtain

$$
\begin{aligned}
& \underline{E}_{2} X=\frac{u}{x}+\frac{v}{y} \\
& \bar{E}_{2} X=\frac{1}{x}+\frac{1}{y}
\end{aligned}
$$

Clearly, see Example $1, \underline{E}_{2} X \neq \underline{E}_{\wedge} X, \bar{E}_{2} X \neq \bar{E}_{\wedge} X, \underline{E}_{2} X \neq \underline{E}_{\&} X$ and $\bar{E}_{2} X \neq \bar{E}_{\&} X$.

From above example, we know that different quantum implications will lead to different rough approximations. 
Proposition 11. For any binary relation $E, l$-valued set $X$ on $U$ and $i=2,3,4,5$,

$$
\begin{aligned}
& \bar{E}_{i} X=\left(\underline{E}_{i} X^{\perp}\right)^{\perp}, \\
& \underline{E}_{i} X=\left(\bar{E}_{i} X^{\perp}\right)^{\perp} .
\end{aligned}
$$

Proof. It can be obtained from the definition of the dual operator, i.e., Equation (8) $u \hookrightarrow_{i}$ $v=\left(u^{\perp} \rightarrow_{i} v^{\perp}\right)^{\perp}$.

Example 5. See Example 4, we have

$$
\begin{aligned}
\left(\underline{E}_{2} X^{\perp}\right)^{\perp}(x) & =\left(\left(E(x, x) \rightarrow_{2} X^{\perp}(x)\right) \bigwedge\left(E(x, y) \rightarrow_{2} X^{\perp}(y)\right)\right)^{\perp} \\
& =\left(\left(1 \rightarrow_{2} u^{\perp}\right) \bigwedge\left(0 \rightarrow_{2} v^{\perp}\right)\right)^{\perp} \\
& =\left(u^{\perp} \wedge 1\right)^{\perp}=u \vee 0=u \\
\left(\underline{E}_{2} X^{\perp}\right)^{\perp}(y) & =\left(\left(E(y, x) \rightarrow_{2} X^{\perp}(x)\right) \bigwedge\left(E(y, y) \rightarrow_{2} X^{\perp}(y)\right)\right)^{\perp} \\
& =\left(\left(0 \rightarrow_{2} u^{\perp}\right) \bigwedge\left(u \rightarrow_{2} v^{\perp}\right)\right)^{\perp} \\
& =\left(1 \wedge v^{\perp}\right)^{\perp}=0 \vee v=v \\
\left(\bar{E}_{2} X^{\perp}\right)^{\perp}(x) & =\left(\left(E(x, x)^{\perp} \hookrightarrow_{2} E^{\perp}(x)\right) \bigvee\left(E(x, y)^{\perp} \hookrightarrow_{2} E^{\perp}(y)\right)\right)^{\perp} \\
& =\left(\left(1^{\perp} \hookrightarrow_{2} u^{\perp}\right) \bigvee\left(0^{\perp} \hookrightarrow_{2} v^{\perp}\right)\right)^{\perp} \\
& =\left(u^{\perp} \vee 0\right)^{\perp}=u \wedge 1=u \\
\left(\bar{E}_{2} X^{\perp}\right)^{\perp}(y) & =\left(\left(E(y, x)^{\perp} \hookrightarrow_{2} E^{\perp}(x)\right) \bigvee\left(E(y, y)^{\perp} \hookrightarrow_{2} E^{\perp}(y)\right)\right)^{\perp} \\
& =\left(\left(0^{\perp} \hookrightarrow_{2} u^{\perp}\right) \bigvee\left(u^{\perp} \hookrightarrow_{2} v^{\perp}\right)\right)^{\perp} \\
& =\left(0 \vee v^{\perp}\right)^{\perp}=1 \wedge v=v
\end{aligned}
$$

It is easy to verify that $\bar{E}_{2} X=\left(\underline{E}_{2} X^{\perp}\right)^{\perp}$ and $\underline{E}_{2} X=\left(\bar{E}_{2} X^{\perp}\right)^{\perp}$.

Proposition 12. There is orthomodular lattice $L$, such that $(v \& u) \vee(w \& u)=(v \vee w) \& u$ holds for any $u, v, w \in L$, and $\underline{E}_{i}(X \cap Y) \neq\left(\underline{E}_{i} X \cap \underline{E}_{\&} Y\right)$ and $\bar{E}_{i}(X \cup Y) \neq\left(\bar{E}_{i} X \cup \bar{E}_{\&} Y\right)$ for some $l$-valued relation $E$ and $l$-valued sets $X$ and $Y$.

Proof. Consider the orthomodular lattice MO2 in Figure 1. Clearly, $(v \& u) \vee(w \& u)=$ $(v \vee w) \& u$ hold in MO2 for any $u, v, w \in M O 2$. Let $E\left(x, y_{1}\right)=u$, and $E(x, y)=0$ for other $y \in U ; X\left(y_{1}\right)=v, Y\left(y_{1}\right)=u^{\perp}$, and $X(y)=Y(y)=0$ for other $y \in U$. Then, we have

$$
\begin{aligned}
E_{2}(X \wedge Y)(x) & =\bigvee_{y \in U}\left(E(x, y) \rightarrow_{2}(X \wedge Y)(y)\right) \\
& =E(x, y) \rightarrow_{2}(X \wedge Y)(y) \\
& =u \rightarrow_{2}\left(v \wedge u^{\perp}\right) \\
& =u \rightarrow_{2} 0 \\
& =u^{\perp} .
\end{aligned}
$$


and

$$
\begin{aligned}
\left(\underline{E}_{2} X \wedge \underline{E}_{2} Y\right)(x) & =\underline{E}_{2} X(x) \wedge \underline{E}_{2} Y(x) \\
& =\left(\bigvee_{y \in U}\left(E(x, y) \rightarrow_{2} X(y)\right)\right) \wedge\left(\bigvee_{y \in U}\left(E(x, y) \rightarrow_{2} Y(y)\right)\right) \\
& =\left(E\left(x, y_{1}\right) \rightarrow_{2} X\left(y_{1}\right)\right) \wedge\left(E\left(x, y_{1}\right) \rightarrow_{2} Y\left(y_{1}\right)\right) \\
& =\left(u \rightarrow_{2} v\right) \wedge\left(u \rightarrow_{2} u^{\perp}\right) \\
& =v \wedge u^{\perp} \\
& =0 .
\end{aligned}
$$

Moreover, we have

$$
\begin{aligned}
& \underline{E}_{3}(X \wedge Y)(x)=u \rightarrow_{3} 0=u^{\perp}, \\
& \underline{E}_{4}(X \wedge Y)(x)=u \rightarrow_{4} 0=u^{\perp}, \\
& \underline{E}_{5}(X \wedge Y)(x)=u \rightarrow_{5} 0=u^{\perp} .
\end{aligned}
$$

and

$$
\begin{aligned}
& \left(\underline{E}_{3} X \wedge \underline{E}_{3} Y\right)(x)=\left(u \rightarrow_{3} v\right) \wedge\left(u \rightarrow_{3} u^{\perp}\right)=0 \wedge u^{\perp}=0, \\
& \left(\underline{E}_{4} X \wedge \underline{E}_{4} Y\right)(x)=\left(u \rightarrow_{4} v\right) \wedge\left(u \rightarrow_{4} u^{\perp}\right)=v^{\perp} \wedge u^{\perp}=0, \\
& \left(\underline{E}_{5} X \wedge \underline{E}_{5} Y\right)(x)=\left(u \rightarrow_{5} v\right) \wedge\left(u \rightarrow_{5} u^{\perp}\right)=u \wedge u^{\perp}=0 .
\end{aligned}
$$

Therefore, $\underline{E}_{i}(X \cap Y) \neq\left(\underline{E}_{i} X \cap \underline{E}_{i} Y\right), i=2,3,4,5$. Then, we can obtain $\bar{E}_{i}(X \cup Y) \neq$ $\left(\bar{E}_{i} X \cup \bar{E}_{i} Y\right), i=2,3,4,5$ from Proposition 11.

From above result, the distributive law of $\vee$ over \& in $\rightarrow_{i}(i=2, \ldots, 5)$ based rough approximations does not play the same part as in $\rightarrow_{1}$ based rough approximations.

\section{Conclusions}

In this paper, we redefined COL-valued rough approximations based on quantum implication. First, COL-valued rough approximations are defined by using the multiplication (\&) and Sasaki implication $\rightarrow_{1}$ instead of meet $\wedge$ and join $\vee$, respectively. This leads to new results that only rely on the distributive law of $\vee$ over \&, which is strictly weaker than the distributive law of $\vee$ over $\wedge$. This is very important for COL-valued rough approximations since similar results in our previous work [26,27] rely on the distributive law of $\vee$ over $\wedge$. So the new quantum rough model needs weaker condition, and is applicable to a bigger extension. We further establish rough approximations via other four quantum implication, which are different from rough approximations via Sasaki implication. Some properties of rough approximations via these four quantum implications do not rely on the distributive law of $\vee$ over \&.

Obviously, underlying rules play an important part in the concept of rough approximations. Some fundamental properties of rough approximations can not hold without some underlying rules of logics. By setting the equivalence between underlying rules of logics and properties of rough approximations, rough models with different ranges can be established. Obviously, the results presented in this paper only considered the distributivity. As future work, we can consider the equivalence between properties of rough approximations and other underlying rules, such as modularity and compatibility in a COL and cancellation law in an effect algebra, which is an algebraic model of unsharp quantum logic [39]. Naturally, a more detailed discussion of other algebraic models and other quantum logics, such as quasi-MV algebras [40,41], quantum MV algebras [42] and quantum computational logics [43], will be both necessary and interesting.

The purpose of this paper and author's previous works [26,27] is to establish a theory of rough sets based on quantum logic. However, as mentioned in [44], quantum logic seems to have no obvious links to quantum computation. The issue how to combine rough set method with quantum computation should be further investigated. 
Funding: This research was funded by the National Science Foundation of China under Grant No. 62006168 and Zhejiang Provincial Natural Science Foundation of China under Grant No. LQ21A010001.

Institutional Review Board Statement: Not applicable.

Informed Consent Statement: Not applicable.

Data Availability Statement: Not applicable.

Conflicts of Interest: The author declares no conflict of interest.

\section{References}

1. Pawlak, Z. Rough sets. Int. J. Comput. Inf. Sci. 1982, 11, 341-356. [CrossRef]

2. Rauszer, C. An equivalence between theory of functional dependence and a fragment of intuitionistic logic. Bul. Pol. Acad. Sci. Math. 1985, 33, 571-579.

3. Vakarelov, D. A modal logic for similarity relations in Pawlak knowledge representation systems. Fundam. Inform. 1991, 15, 61-79. [CrossRef]

4. Vakarelov, D. Modal logics for knowledge representation systems. Theor. Comput. Sci. 1991, 90, $433-456$.

5. Yao, Y.Y. Constructive and algebraic methods of the theory of rough sets. Inf. Sci. 1998, 109, 21-47. [CrossRef]

6. Pei, Z.; Pei, D.; Zheng, L. Topology vs. generalized rough sets. Int. J. Approx. Reason. 2011, 52, 231-239. [CrossRef]

7. Pawlak, Z.; Skowron, A. Rough sets and boolean reasoning. Inf. Sci. 2007, 177, 41-73. [CrossRef]

8. Qi, G.; Liu, W. Rough operations on Boolean algebras. Inf. Sci. 2005, 173, 49-63. [CrossRef]

9. Hua, X. Fuzzy rough set based on lattice effect algebra. J. Intell. Fuzzy Syst. 2019, 36, 29-36. [CrossRef]

10. Radzikowska, A.M.; Kerre, E.E. Fuzzy rough sets based on residuated lattices. Trans. Rough Sets Lect. Notes Comput. Sci. 2004, 3135, 278-296.

11. Bao, Y.; Yang, H.; She, Y. Using one axiom to characterize L-fuzzy rough approximation operators based on residuated lattices. Fuzzy Sets Syst. 2018, 336, 87-115. [CrossRef]

12. Yin, Y.Q.; Huang, X.K. Fuzzy Roughness in hyperrings based on a complete residuated lattice. Int. J. Fuzzy Syst. 2010, 13, 185-194.

13. Yin, Y.Q.; Zhan, J.M.; Corsini, P. Fuzzy roughness of n-ary hypergroups based on a complete residuated lattice. Neural Comput. Appl. 2011, 20, 41-57. [CrossRef]

14. Wang, C.Y.; Hu, B.Q. Fuzzy rough sets based on generalized residuated lattices. Inf. Sci. 2013, 248, 31-49. [CrossRef]

15. She, Y.H.; Wang, G.J. An axiomatic approach of fuzzy rough sets based on residuated lattices. Comput. Math. Appl. 2009, 58, 189-201. [CrossRef]

16. Chen, D.; Zhang, W.; Yeung, D.; Tsang, E.C.C. Rough approximations on a complete completely distributive lattice with applications to generalized rough sets. Inf. Sci. 2006, 176, 1829-1848.

17. Liu, G.L. Generalized rough sets over fuzzy lattices. Inf. Sci. 2008, 178, 1651-1662. [CrossRef]

18. Ma, L.W. Two fuzzy covering rough set models and their generalizations over fuzzy lattices. Fuzzy Sets Syst. 2016, $294,1-17$. [CrossRef]

19. Feynman, R.P. Simulating physics with computers. Int. J. Theor. Phys. 1982, 21, 467-488. [CrossRef]

20. Feynman, R.P. Quantum mechanical computers. Found. Phys. 1986, 16, 507-531. [CrossRef]

21. Deutsch, D. Quantum theory, the Church-Turing principle and the universal quantum computer. Proc. R. Soc. Lond. A 1985, 400, 97-117.

22. Shor, P.W. Algorithms for quantum computation: Discrete logarithms and factoring. In Proceedings of the 35th Annual Symposium on Foundations of Computer Science, Santa Fe, NM, USA, 20-22 November 1994; IEEE Press: Los Alamitos, CA, 1994; pp. 124-134.

23. Grover, L.K. A fast quantum mechanical algorithm for database search. In Proceedings of the 28th Annual ACM Symposium on the Theory of Computing, Philadelphia, PA, USA, 22-24 May 1996; p. 212.

24. Birkhoff, G.; von Neumann, J. The logic of quantum mechanics. Ann. Math. 1936, 37, 823-843. [CrossRef]

25. Hassan, Y.F. Rough set classification based on quantum logic. J. Exp. Theor. Artif. Intell. 2017, 29, 1325-1336. [CrossRef]

26. Dai, S. Topological characterizations of rough set theory based on quantum logic. New Math. Natural Comput. 2021. [CrossRef]

27. Dai, S. Rough Approximation Operators on a Complete Orthomodular Lattice. Axioms 2021, 10, 164. [CrossRef]

28. Pták, P.; Pulmannová, S. Orthomodular Structures as Quantum Logics; Kluwer: Dordrecht, The Netherland, 1991.

29. Mittelstaedt, P. Quantum Logic; D. Reidel Publ. Co.: Dordrecht, The Netherland, 1978.

30. Ying, M.S. Automata theory based on quantum logic(II). Int. J. Theoret. Phys. 2000, 39, 2545-2557. [CrossRef]

31. Ying, M.S. A theory of computation based on quantum logic(I). Theor. Comput. Sci. 2005, 344, 134-207. [CrossRef]

32. Qiu, D.W. Automata theory based on quantum logic: Some characterizations. Inf. Comput. 2004, 190, 179-195. [CrossRef]

33. Dai, S. A note on implication operators of quantum logic. Quantum Mach. Intell. 2020, 2, 15. [CrossRef]

34. Davey, B.A.; Priestley, H.A. Introduction to Lattices and Order; Cambridge University Press: Cambridge, UK, 2002.

35. Chiara, M.L.D. Quantum logic. In Handbook of Philosophical Logic; Gabbay, D., Guenthner, F., Eds.; Reidel: Dordrecht, The Netherland, 1986; Volume III, pp. 427-469. 
36. Kalmbach, G. Orthomodular lattices. In London Math Soc Monographs; Academic Press: London, UK, 1983.

37. Finch, P.D. Quantum logic as an implicatiom algebra. Bull. Austral. Math. Soc. 1970, 2, 101-106. [CrossRef]

38. Román, L.; Rumbos, B. Quantum logic revisited. Found. Phys. 1991, 21, 727-734. [CrossRef]

39. Chiara, M.L.D.; Giuntini, R.; Greechie, R. Reasoning in Quantum Theory: Sharp and Unsharp Quantum Logics; Springer: Dordrecht, The Netherlands, 2004.

40. Dai, S. Quasi-MV algebras for complex fuzzy logic. AIMS Math. 2022, 7, 1416-1428. [CrossRef]

41. Ledda, A.; Konig, M.; Paoli, F.; Giuntini, R. MV algebras and quantum computation. Stud. Logica 2006, 82, 245-270. [CrossRef]

42. Giuntini, R. Quantum MV algebras. Stud. Logica 1996, 56, 393-417. [CrossRef]

43. Gudder, S. Quantum Computational Logic. Int. J. Theor. Phys. 2003, 42, 39-47. [CrossRef]

44. Ying, M.S. Automata Theory Based on Quantum Logic(I). Int. J. Theor. Phys. 2000, 39, 985-995. 